\title{
Posibilidades didácticas del sonido y radio escolar: Implicaciones curriculares
}

\author{
Isidro Moreno
}

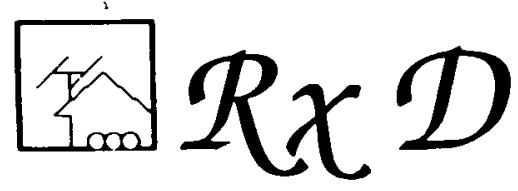

La radio es uno de los medios tecnológicos más accesibles sin grandes recursos al medio escolar, a la vez que brinda un repertorio didáctico enormemente rico y flexible. En este artículo se pasa revista a un buen abanico de estas posibilidades.

\section{¿POR QUE LA RADIO?}

Porque es magia y magia tiene mucho que ver con imaginación. De esta forma obtenemos IMAGINACION, que es casi, casi imagen en acción. $Y$ esto es lo que hace la radio, poner en marcha la imaginación.

Me gustaría intercalar con estas palabras música de fondo. Aquí haríamos una pausa y pasaríamos la música a primer plano...

$\mathrm{Y}$ es que la radio es música también. En realidad es la magia del sonido. El sonido se hace lenguaje, ya lo sabemos. Y mira tú por dónde, en la radio aglutinamos muchos lenguajes. Hasta el icónico, es decir, la imagen. De una forma fenomenal, puesto que cada uno se inventa su imagen.

Como educadores sensibilizados en los temas de los mass media, creo que debemos interesarnos en la investigación sobre el impacto del universo informativo en la escuela y las implicaciones pedagógicas que éste conlleva.

Hemos dicho hace un momento que la radio aglutina lenguajes y entre ellos el más usado, el verbal. La palabra va a dar cuerpo al mensaje que configura la comunicación entre el emisor y el oyente. Recordemos que la educación es, o debe ser, un acto de comunicación. La palabra, que incluso etimológicamente es parábola - parabolé- es decir, alegoría o comparación, tiene un sentido mágico al igual que la radio. Todas estas connotaciones, esos significados de que es capaz la palabra, facilitan ese acto maravilloso que es hablar, comunicar, comunicarse...

Y la palabra va y viene por el aire, como el rayo de luz..., el rayo, radium. La radio otra vez... Pero esto no es una lección de etimología; he pretendido 
enfocar el tema de la radio desde una perspectiva digamos que mágica. Otra vez la magia.

Sí quiero hacer una reflexión, o mejor dicho un apunte, un boceto de reflexión. Para ello vamos a sustituir el vocablo «radio» por un término más amplio: Posibilidades del, con, desde... el sonido. Esta «amplitud» nos va a permitir más «longitud» para conseguir una «frecuencia» - más o menos moduladaacerca de estas posibilidades. Ya ves que sin querer estamos jugando con las coordenadas de propagación del sonido... la palabra, las connotaciones, la magia.

\section{APUNTES PARA UNA REFLEXION}

Las posibilidades didácticas que mencionábamos anteriormente las podemos encauzar desde tres puntos de vista, o desde tres ejes que nos van a permitir su estudio, pero que, evidentemente, están interrelacionados. Estos tres enfoques serían:

El sonido - la radio- como:

- Instrumento y recurso.

- Medio de expresión y comunicación.

- Análisis crítico de la información.

Conviene aclarar también la situación, desde el punto de vista de la teoría curricular, desde la que hacemos esta reflexión. De acuerdo con la descripción que hacen Carr y Kemmis (1988), nuestro planteamiento lo ubicamos desde la teoría del currículum práctico o interpretativo. Decimos «desde», porque el objetivo final será instalarnos, en mayor o menor medida, en el uso de los medios desde una perspectiva estratégica y crítica, capaz de transformar y modificar los códigos siempre que éstos supongan diferencias sociales y culturales.

Recientemente, A. Bautista (1989) contempla tres tipos de uso de los medios siguiendo precisamente estos tres modelos del currículum. Un uso transmisor-reproductor orientado por la teoría curricular técnica o tecnología cuyas características principales serían la linealidad en el diseño, la escasa adecuación a la realidad y el papel reproductor y ejecutor del maestro de las orientaciones que le llegan diseñadas.

Un segundo uso práctico-situacional según el modelo curricular práctico o interpretativo, caracterizado, entre otras cosas, por el análisis de la realidad para dar significado a todas las situaciones, diseños abiertos que tienen en cuenta esa realidad y utilización recursiva de los medios que permite aprender y utilizar sistemas de representación para desarrollar el pensamiento, solucionar problemas e interpretar y relacionarse con el medio físico, social y cultural.

Por último, la teoría crítica del currículum inspiraría el uso crítico, transformador, en donde los medios serían utilizados como elementos de análisis y reflexión sobre la práctica con el fin de transformarla y mejorarla.

Aclarada pues nuestra postura, vamos a desarrollar esa triple vertiente que anunciábamos en cuanto a las posibilidades didácticas del sonido.

\section{a) El sonido como instrumento.}

En este punto hacemos referencia a la utilización del medio sonoro - tanto desde el punto de vista del material de equipo como del material de paso elaborado por nosotros mismos - como un recurso a utilizar en distintos momentos 
de nuestra práctica docente. Así nos serviremos de él como instrumento motivador en la presentación de algunos temas, ya sea con grabaciones originales, ya con grabaciones que nosotros o los alumnos hayamos elaborado. La grabación de un momento de nuestra actuación puede servirnos de autodiagnóstico de destrezas; así como la grabación de un debate o asamblea de clase permite a los alumnos oírse y analizar cómo son sus intervenciones y actitudes de escucha.

Aprender a escuchar o a mejorar el hábito de escucha puede ser uno de los grandes objetivos de utilización de este medio. Para ello podemos disponer sencillos ejercicios como son: averiguar cuántas veces se repite determinada palabra; identificar distintos sonidos; seguir una serie de instrucciones; utilizar el contexto del discurso explicando el significado de palabras poco conocidas; reconstruir una historia a partir de efectos sonoros descolocados; etcétera.

En el ámbito de la expresión oral, el trabajo de dicción y expresividad de la voz constituye, asimismo, otra gran actividad que nos permite abordar algunos de los objetivos propuestos en el Diseño Curricular Base del área de Lengua y Literatura.

\section{b) El sonido como medio de expresión y comunicación.}

En la introducción al DCB de Lengua y Literatura, tanto en educación Primaria como en Secundaria, se hace mención al desarrollo «de la capacidad de utilizar el lenguaje como instrumento de comunicación y de representación, y de conocimiento». (...) «Aprender la lengua es aprender a usarla, manipularla, crearla y recrearla» $(1989,261)$. El medio que nos ocupa puede servir de soporte para la materialización del enfoque instrumental y funcional que se nos hace en la nueva propuesta.

En las orientaciones didácticas y para la evaluación, cuando nos plantean el análisis y reflexión sobre la lengua, podemos leer: «Esta opción sólo tiene sentido dentro de un planteamiento global de la enseñanza de la lengua» (...) «... el trabajo de vocabulario y de estructuras de la lengua siempre irá asociado a las situaciones de comunicación oral y escrita que se planteen en el aula; lo mismo sucede con aspectos como la ortografía» (1989, 309-310).

En realidad, es bien poco lo que podemos encontrar referido al sonido tal y como nosotros lo entendemos. No obstante, si planteamos su utilización desde una perspectiva práctica e interpretativa, muy bien puede concretarse nuestro enfoque en alguna de las sugerencias sobre recursos didácticos, pues «... el profesor debe aceptar que, en la actualidad, las nuevas tecnologías aportan medios y productos cada vez más atractivos para la juventud». Por tanto «El aula ha de ser un lugar en el que el adolescente tenga algo que decir y en el que su palabra sea tenida en cuenta, de manera que participe, opine, coopere, debata sus ideas y valores, etc. (...) Conviene que existan espacios apropiados (dentro y fuera del aula) que permitan y fomenten las diferentes actividades y en las que se encuentren fácilmente los materiales necesarios: biblioteca de aula y centro, archivos, mediateca, laboratorio de sonido e imagen, etcétera.» (1989-404-405).

Así pues, esta perspectiva nuestra sobre el sonido nos permite desarrollar contenidos conceptuales, de procedimientos y valores a través de sencillas actividades como son audiciones, creación de textos y programas, elaboración de noticias, grabaciones, etc., en cualquiera de las áreas que estructuran las distintas etapas. 


\section{c) Análisis crítico de la información.}

La rapidez con que hoy día se sucede todo, los cambios y transformaciones a que nos vemos sometidos que alteran, incluso, nuestra manera de vivir y entender el mundo, la sobredosis de información que nos atrapa en la maravillosa red de la electrónica, hacen de nosotros «seres inadecuados». Nuestra obligación como profesionales de la educación es estar al «loro» (lo dicen los chavales), sobre todo si queremos «conectar» con la realidad que en clase se nos presenta todos los días. Precisamente por eso, porque esa realidad cotidiana de seres inadecuados a la vertiginosa realidad de fuera de la escuela necesita de un modelo crítico, de un instrumento que la capacite para analizar, descodificar y entender los múltiples mensajes.

Aquí encontramos, por fin, nuestra tercera vertiente. Se trata de analizar críticamente la información que nos llega. Volvemos a leer en las orientaciones didácticas del DCB de Lengua y Literatura: «Ante la insistente presencia de los medios de comunicación de masas, el niño, el joven e, incluso, el adulto, se enfrentan a discursos nuevos, muchas veces opuestos a aquellos con los que han sido educados. Los actuales aprendizajes deben tener en cuenta no sólo la realidad, sino también la interpretación que otros ofrecen de esa realidad» (1989, 423). Asimismo, este análisis es susceptible de ser tratado en otras áreas, y en los niveles educativos más bajos puede iniciar todo un proyecto de trabajo globalizado.

A través de pequeñas pautas de análisis, sencillas escalas de observación que nosotros mismos podemos construir guías que nos permitan comparar, podemos ir elaborando nuestros propios instrumentos al tiempo que diseñamos distintas actividades que desarrollen el conocimiento y estudio crítico de la información que nos llega. Conocer el proceso de elaboración de la noticia, los recursos que se emplean, los lenguajes radiofónicos, puede convertirse en una aventura apasionante.

Este análisis, esta descodificación, este desengranaje deben servir para que, a medida que aumenta el conocimiento, se pongan en marcha los mecanismos psicológicos de representación y utilización de otros códigos como apuntábamos en el apartado anterior. Como educadores tenemos la obligación de adecuar a esos «seres inadecuados» que decíamos al principio al medio que nos rodea para transformarlo, e intentar que éste sea más humanizado, y esto sólo es posible desde la autonomía personal, la solidaridad, la comprensión...

\section{TRES EJEMPLOS}

Con el fin de ilustrar cada uno de los enfoques anteriores quiero presentar tres experiencias realizadas con mis alumnos y alumnas. Por razones de espacio, presento únicamente el planteamiento y posibilidades de cada una de éstas.

\section{a) El sonido como instrumento: Recurso para la autocorrección de la lectura.}

Desde mi privilegiada posición de profesor de Lengua he podido poner en práctica muchas de las ideas, intuiciones y experiencias relacionadas con el mundo del sonido, la radio y, en general, los medios y recursos audiovisuales.

Una actividad que desde siempre ha presidido nuestras clases ha sido la lectura, la lectura comprensiva evidentemente, pero también el «buen leer». La re- 
citación, el leer ante un público, en este caso la clase, permite a los chicos y a la chicas hacer un esfuerzo por pronunciar mejor, hacer la pausas oportunas, enfatizar algunos pasajes importantes, descubrir el ritmo del discurso, comprender mejor el texto y disfrutar del placer de leer.

Así pues, el recitado, ha sido una actividad sistemática en nuestras clases de lengua. El desarrollo de ésta es muy sencillo; los alumnos eligen un pequeño texto, un poema o la letra de alguna canción. Quincenalmente hay una sesión de lectura o recitado y son dos alumnos los que leen. La clase sufre también una pequeña transformación, hay que prepararla para el acontecimiento con alguna luz, velas, música y el atril.

Al tiempo que los alumnos leen, se graba en un magnetófono lo que dicen. Después hay una puesta en común en donde el resto de la clase hace una crítica, en base a unos criterios, sobre cómo han leído. Posteriormente, los que han leído se llevan la grabación a su casa y se oyen con el fin de autocorregirse; para ello disponen de una ficha guía en donde van anotando lo que ellos perciben. Esta ficha permite ver el perfil lector y los avances o retrocesos.

En general, he de decir que este método ha dado resultados bastante satisfactorios. Como se ve es una utilización sencilla de un instrumento, el magnetófono, que hoy está al alcance de cualquiera y es raro el centro educativo que carece de este tipo de aparatos.

\section{b) El Sonido como medio de expresión y comunicación: Géneros periodísticos.}

Cada día es más frecuente el trabajo con la prensa escrita en las aulas; quizá no lo es tanto el abordar los temas desde un punto de vista radiofónico. Sin despreciar en absoluto el primero, es más, sirviéndonos del periódico, podemos organizar la información que éste nos proporciona para elaborar mensajes orales, información radiofónica, boletines informativos o como los queramos llamar.

La diferencia con la información escrita es evidente, ahora hay que escribir para hablar, lo que implica una gran capacidad de síntesis (no conviene ser pesado) y utilizar un vocabulario sencillo, asequible y que cuente lo que queremos que cuente. El mensaje radiofónico es efímero, es necesario utilizar la reiteración, pero en su justa medida.

Como géneros periodísticos abordamos en esta experiencia los siguientes: la crónica, el reportaje y la mesa redonda o tertulia.

Para elaborar las distintas informaciones nos servimos de recortes de periódicos: titulares y alguna entradilla.

La crónica permite hacer un trabajo individual en donde el alumno-periodista nos cuenta, nos informa acerca de acontecimientos sin entrar en valoraciones personales. Puede ajustar su relato a hechos reales o, por el contrario, puede inventar la historia. El objetivo del trabajo es hacer una crónica, no lo olvidemos.

El reportaje se plantea como trabajo en pequeños grupos. Así como la crónica tiene un carácter puntual, el reportaje es más bien intemporal. El grupo debe informarse sobre el tema acudiendo a libros, artículos y reportajes para elaborar su información. Pueden introducir sus puntos de vista y orientar la información en el sentido que deseen.

En la mesa redonda o tertulia abordamos temas de opinión. Es también un trabajo en equipo pero, a diferencia del anterior, existe un momento de preparación individual de forma que cada alumno pueda tener una opinión personal sobre el caso que se plantea. Posteriormente se entra en debate; alguien presen- 
ta el caso y hace las veces de moderador, resumiendo al final la opinión u opiniones vertidas a lo largo de la tertulia.

Con estas tres actividades ocupamos perfectamente a todo un grupo de alumnos y alumnas. Una vez hechos todos los preparativos se realizan las correspondientes grabaciones, teniendo en cuenta las normas de escribir para habiar, la música que se va a emplear (previamente seleccionada) y elaborando un pequeño guión técnico que nos permite ordenar todos los elementos sonoros: vozpalabra, música, ráfagas musicales, efectos, etc. $\mathrm{Si}$, como es nuestro caso, se tiene la oportunidad de emitir desde la emisora escolar, pues tanto mejor; si no es así, las grabaciones se oyen en clase, en la megafonía interior del centro, e incluso cabe la posibilidad de, al tiempo que se graba, simular en la propia clase un estudio de radio. La audición posterior puede convertirse también en instrumento de valoración, como veíamos en el apartado anterior; a tal efecto, disponemos de hojas de observación.

\section{c) Análisis crítico de la información: Análisis comparativo de informativos.}

Conocer quién nos cuenta la noticia nos permite saber qué hay detrás de ella, qué intenciones, por qué así y no de otra manera. El material que utilizamos en esta ocasión es material grabado directamente de la radio. Para ello previamente hemos grabado dos espacios informativos de dos emisoras diferentes. Por otra parte, quien realice este tipo de actividad debe tener un cierto conocimiento del ámbito informativo y de lo que hay detrás de las empresas que nos suministran la información en general.

Tener la información grabada nos permite oírla cuantas veces haga falta. Es una actividad para trabajar en equipo y que desarrollamos de la siguiente manera:

A cada equipo entregamos un esquema de «Recursos informativos» que explicamos detenidamente (figura 1). Se suministran además dos modelos de fichas que son las que van a permitir hacer el análisis comparativo (figuras 2 y 3 ).

En primer lugar se hace una audición (puede repetirse las veces que haga falta) de los sumarios respectivos de ambos informativos, al mismo tiempo se toman notas. Se cumplimenta la primera ficha. Cada equipo elige una noticia, procurando que la misma se haya dado en los dos sumarios.

Audición del desarrollo de la noticia elegida, los integrantes del equipo toman notas, miden el tiempo, etc., y cumplimentan la ficha número dos. Entre todos sacan conclusiones, las escriben y se presenta todo el material en la puesta en común final.

\section{CONCLUSIONES}

En otro momento hablaremos de lo específico de la radio escolar, desde el punto de vista técnico y pedagógico. Sirva como anticipo y a modo de conclusión esta somera explicación del esquema siguiente:

En cuanto a la utilización del medio sonoro ya hemos visto el triple planteamiento del que partimos: como instrumento-recurso, como medio de expresión y comunicación y como análisis crítico de la información. Conviene, no obstante, recordar que nuestro planteamiento lo hacemos desde la perspectiva curricular práctica o interpretativa que nos va a permitir elaborar nuestro propio material, 
Figura 1

Esquema de recursos informativos

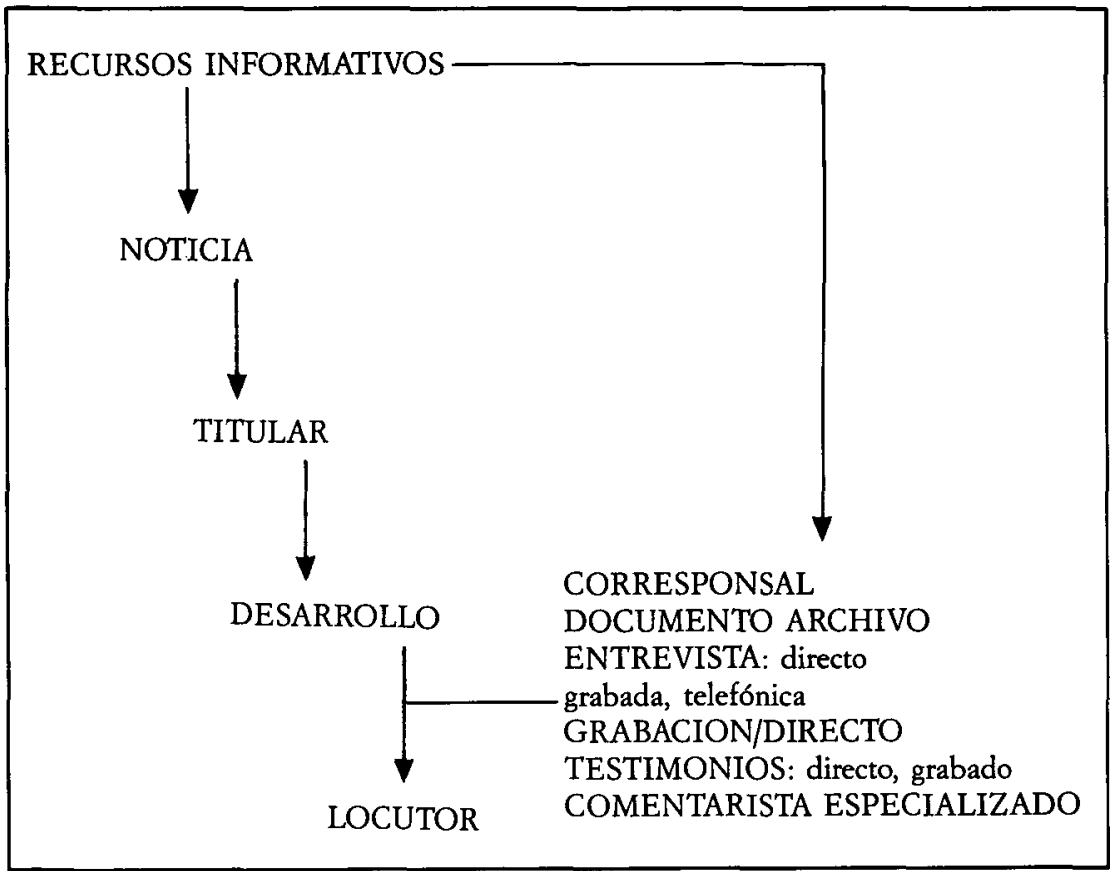

FIGURA 2

INFORMATIVOS: ANALISIS COMPARATIVO

Ficha de análisis comparativo de informativos

HORA:

DIA:

EMISORA

EMISORA

PROGRAMA:

PROGRAMA:

\begin{tabular}{cccc}
\hline \multicolumn{2}{c}{ SUMARIO } & \multicolumn{2}{c}{ SUMARIO } \\
\hline \multirow{2}{*}{ TITULARES } & RECURSOS & TITULARES & $\begin{array}{c}\text { RECURSOS } \\
\text { SONOROS }\end{array}$ \\
& SONOROS & & \\
\hline
\end{tabular}

FIgURA 3

ANALISIS COMPARATIVO: DESARROLLO DE UNA NOTICIA

Ficha de análisis comparativo de desarrollo de una noticia

TITULAR:

PROGRAMA: PROGRAMA:

DESARROLLO TIEMPO RECURSOS DESARROLLO TIEMPO RECURSOS




\section{4}

dotarnos de instrumentos para la resolución de problemas, aplicar a la realidad nuestra actuación y, entre otras cosas, reflexionar sobre la práctica.

Desde el punto de vista de la actividad, existe una doble vertiente: lo estrictamente radiofónico y lo pedagógico. Es decir, puede que nos interese la utilización del medio sonoro exclusivamente como actividad pedagógica. Entraríamos en el ámbito de la expresión oral y escrita, la mejora de hábitos de escucha y participación, etc. Si nuestro interés es doble, tendríamos que hacer referencia al lenguaje radiofónico: palabra, música, sonido, silencio. Actividades que encajan perfectamente en la clase de Lengua, en el área Artística e incluso en otras.

Quedarían, finalmente, algunas actividades radiofónicas, quizá más propias de un taller, como son algunas de las que se enumeran en la figura 4.

No olvidemos, por último, que todo este planteamiento no se puede dar aislado. Debe estar inmerso a lo largo de todo el proceso de enseñanza y de aprendizaje. No debe convertirse en la anécdota puntual y graciosa que un buen día realizamos. Forma parte del currículum y como tal hemos de contemplarlo, unas veces como procedimientos para desarrollar ciertas habilidades y estrategias didácticas, otras veces como adquisición de conceptos $\mathrm{y}$, por supuesto, siempre como desarrollo de actitudes y valores.

FIGURA 4

\section{RADIOFONICAS \\ PEDAGOGICAS}

MANEJO DE APARATOS

LOCUCION

GUIONIZACION

PRODUCCION

EMISION-GRABACION

DOCUMENTACION

INFORMACION

AUDICIONES

CREACION EMISORA

ESCOLAR

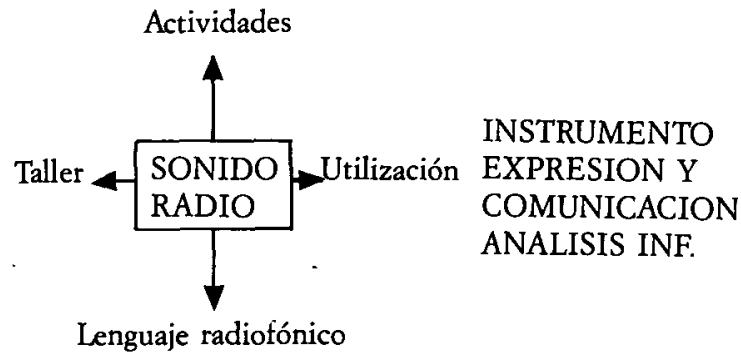

PALABRA

MUSICA

SONIDO

SILENCIO 


\section{Referencias}

BaUtista, A. (1989): «El uso de los medios desde los modelos del currículum», en Comunicación, Lenguaje y Educación. Madrid: Aprendizaje, 3-4, 39-51.

BurRiel, J. M. a. (1981): El Reto de las Ondas. Barcelona: Salvat. Col.: Temas clave.

CarR, W. y Kemmis, S. (1988): Teoría Crítica de la enseñanza. Barcelona: Martínez Roca.

Cebrián Herreros, M. (1988): Teoría y técnica de la información audiovisual. Madrid: Alhambra U.

EMISORES DE FM. n. ${ }^{\circ}$ 2: La Radio a su Alcance. Barcelona: Ingeler.

Forestier, R. (1980): Despertar al Arte. Introducción al Mundo Sonoro. Barcelona: MédicoTécnica.

GonzÁlez I Monge, F. (1989): En el Dial de mi Pupitre. Las Ondas, Herramienta Educativa. Barcelona: G. Gili. Col.: MM. de CC. en la Enseñanza.

Hills, GeORGe (1987): Los Informativos en Radiotelevisión. Madrid: IORT.

MACLEISH, R. (1985): Técnicas de Creación y Realización en Radio. Madrid: IORT.

Manual de Grabación. (1983): Madrid: SM.

Ministerio de Educación. (1989): Diseño Curricular Base de Lengua y Literatura. Madrid: MEC.

MuÑoz, J. J., y GIL, D. (1986): La Radio: Teoría Práctica. Madrid: IORT.

Plana, Gabriel. (1984): Cine: Sonorización. Barcelona: Ins. Parramón.

Registro de Sonido (1986): Biblioteca Básica Electrónica Madrid: Nueva Lente. N. 36.

\section{Posibilidades didácticas del sonido y radio escolar: Implicaciones curriculares. Isidro Moreno. CL\&E, 1992, 14, pp. 57-65}

Resumen: En el presente artículo, el autor, hace una síntesis de los lenguajes radiofónicos desde una perspectiva «mágica» que sirve de introducción a la posterior reflexión en una triple vertiente: El sonido - la radio- como instrumento y recurso, como medio de expresión y comunicacición y como análisis crítico de la información.

Relaciona estos tres enfoques con los modelos de teoría curricular. Asimismo, lo hace con algunos aspectos del Diseño Curricular Base de Lengua y Literatura. Finalmente ilustra su reflexión con tres experiencias llevadas a cabo por el propio autor.

Datos sobre el autor: Maestro y licenciado en Pedagogía. Miembro del movimiento de Renovación Pedagógica «Escuela Abierta» de Getafe. Conocedor de los mass media y sus im. plicaciones didácticas, ha desarrollado experiencias en el aula y en la formación del profesorado, así como en el campo de la Tecnología Educativa. Tiene diversas publicaciones sobre creatividad, expresión y comunicación. Desarrolla su tesis doctoral sobre las claves para formar profesores creativos. Actualmente es director del Centro de Profesores de Getafe.

Dirección: Isidro Moreno Herrero. Centro de Profesores. C/ Pizarro, 21. 2802 Getafe. Madrid.

(C) De todos los artículos deberá solicitarse por escrito autorización de CL\&E y de los autores para el uso en forma de facsímil, fotocopia o cualquier otro medio de reproducción impresa. CL\&E se reserva el derecho de interponer las acciones legales necesarias en aquellos casos en que se contravenga la ley de derechos de autor. 